\title{
CHAPTER 84
}

\section{Santiago de Chile}

\section{Benjamin Kickhöfer and Alejandro Tirachini}

\subsection{Introduction}

This section describes the creation process of the freely available MATSim scenario of Santiago de Chile. The first version of a calibrated scenario is available online ${ }^{1}$ and is documented in Kickhöfer et al. (2016). For the scenario setup, three open data sources are used: (i) car network information from OSM (OpenStreetMap), (ii) PT (Public Transport) supply data from GTFS (General Transit Feed Specification), and (iii) travel diaries from Santiago's 2012 Origin-Destination Survey.

Multiple interventions in Santiago's transport system in the past 20 years make this city an interesting case study for the analysis of alternative transport policies. Santiago has a Metro (subway) network of five lines over 104 kilometers, with two new lines to be launched in 2017 and 2018, adding 37 kilometers to the network. In the city, there is a full-scale integrated public transport system launched in February 2007-the Transantiago system (Muñoz et al., 2014), which has fare integration between all urban buses and the Metro through the use of a single prepaid (smartcard) payment method. There also exists a network of 200 kilometers of tolled urban highways. In winter, the air pollution problem is tackled, in part, by introducing plate-number based car driving bans on the most polluted days. All these elements make Santiago an appealing case study for the application of a metropolitan-scale transport and activity simulator.

1 See https://svn.vsp.tu-berlin.de/repos/public-svn/matsim/scenarios/countries/cl/santiago/ or search from http://matsim.org/datasets.

\section{How to cite this book chapter:}

Kickhöfer, B and Tirachini, A. 2016. Santiago de Chile. In: Horni, A, Nagel, K and Axhausen, K W. (eds.) The Multi-Agent Transport Simulation MATSim, Pp. 491-494. London: Ubiquity Press. DOI: http://dx.doi.org/10.5334/baw.84. License: CC-BY 4.0 


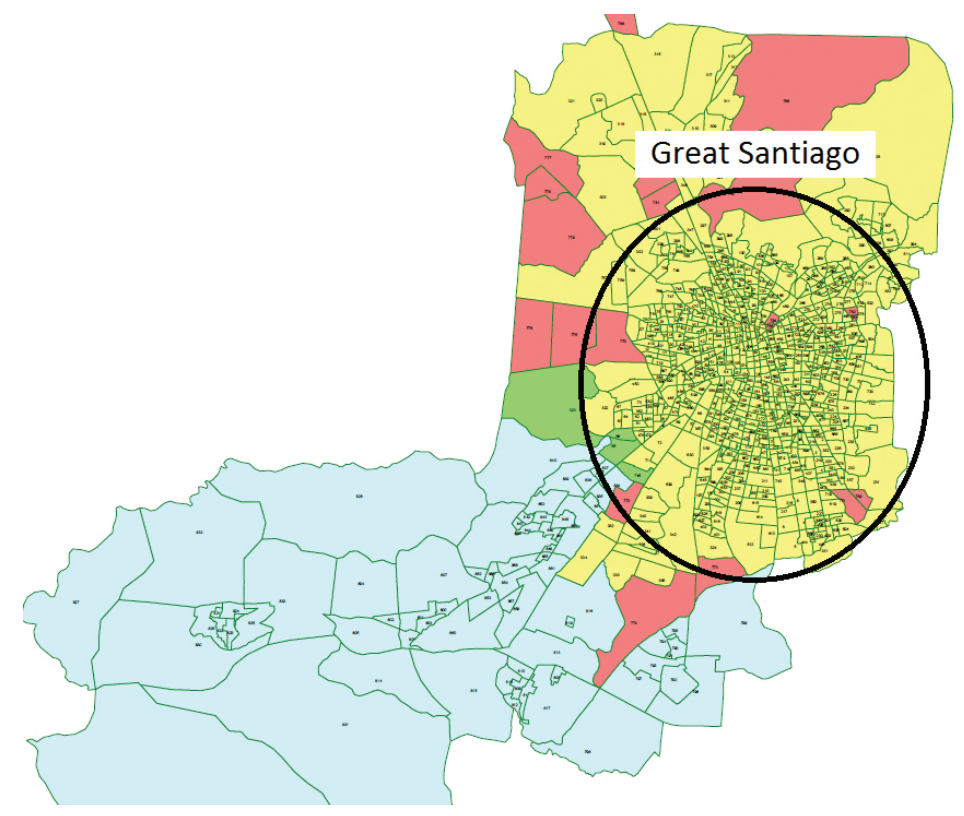

Figure 84.1: 2012 ODS study area and zones, adapted from SECTRA (2014).

\subsection{Data}

\subsubsection{The 2012 origin-destination survey}

The travel demand and activity patterns of the MATSim Santiago scenario are based on the travel and activity data collected in the 2012 Origin-Destination Survey (ODS), whose database and results were released to the public in March 2015. ${ }^{2}$ The surveyed area encompasses 45 comunas (municipalities) of the Santiago Metropolitan Region, with an estimated population of 6.65 million people. The survey goes beyond the Great Santiago Area to include the neighboring municipalities of Colina, Lampa, Pirque, Calera de Tango and Melipilla. The total area has 2 million households with an average of 3.24 persons per household. The sample size is 18000 randomly chosen households along 866 zones that were defined for the survey. Figure 84.1 shows a map of the survey area and zones. The Great Santiago Area is highlighted by an ellipse, in which $91 \%$ of the population is concentrated.

It is estimated that, on a normal working day, there are 18.5 million trips, from which $38.5 \%$ are by non-motorized means (walking and cycling). Around $25 \%$ of the total trips are made using the Transantiago public transport system, out of which $52.4 \%$ are bus-only trips, $22.2 \%$ are metroonly trips and $25.4 \%$ are combined bus-metro trips. Car travel has a modal share of approximately $26 \%$ of the total trips.

In total, 60054 individuals were interviewed in the 2012 ODS, with a total of 113591 trips. Omitting all individuals that do not have two activities plus one connecting trip reduces the sample size to 42459 synthetic agents ( $70.7 \%$ of all interviewees). Therefore, considering the population of the whole metropolitan area of the sample (6.65 million), the MATSim synthetic population represents

\footnotetext{
2 The survey form, reports and full database are available at the website of Chile's Transport Planning Office (SECTRA), http://www. sectra.gob.cl/biblioteca/detalle1 . asp?mfn=3253, accessed 16 August 2015.
} 
approximately a $0.65 \%$ sample, with agents performing activities of the following types: home, at work, work-related, education, health-related, visit someone, shopping, leisure and other.

\subsubsection{Road network and public transport supply}

The source data for the MATSim Santiago road network is taken from OSM. The source data for the Transantiago PT routes and departure times/service frequencies at the stops over time-of-day is a GTFS file 3 , published and continuously updated by Santiago's Metropolitan Public Transport Authority (Directorio de Transporte Público Metropolitano, DTPM). The GTFS file includes all bus and Metro services.

From the MATSim transit schedule, a pseudo transit network is created along with the transit vehicles. This transit network connects-for each transit line-the stops directly to each other. It is not connected to the car network, and only follows the car network's geometry where the resolution of transit stops is high (i.e., where a transit line has a stop at every corner). In consequence, cars and buses run in separate networks; as a result it is currently not possible to analyze, for example, crosscongestion effects between modes. Nonetheless, current congestion patterns of PT are exogenously included, since bus travel times are set to be larger in peak periods, calibrated using historical data from buses that are equipped with GPS devices.

\subsection{Setting up the Open Scenario}

\subsubsection{Scenario specifications}

By converting the input data into MATSim format, several files are generated to run the simulation. Since there are no data restrictions, these files are provided as an open scenario. ${ }^{4}$ The code for obtaining this data from the input data is also publicly available. ${ }^{5}$ Behavioral parameters are taken from a study by Munizaga et al. (2008) and converted into MATSim parameters (Kickhöfer et al., 2016). When performing mode choice, in the present version of the model, agents are only allowed to switch between the transport modes car, PT and walk. Trips performed by any other mode (bike, colectivo, other, ride, taxi, train) remain fixed but can be included in the choice set in future versions. PT captive users are taken into account since agents are only allowed to use a car if they have access to a car according to the survey data. Otherwise their only options are PT and walk. The attributes of the three different modes considered in the present study are travel time (car, PT, walk) and monetary costs (car, PT). Travel time for car trips is a direct output of the simulation where vehicles interact on the road network. Hence, the car travel time also includes road congestion. Travel times for PT results from the GTFS data (station-to-station travel times including transfer time) plus access and egress times done by the walk mode. Hence, the PT travel times do only partly include road congestion, i.e., as long as it is approximated correctly by the schedule, which uses longer travel times in peak periods. Travel times for walk are approximated by teleporting agents between their activities $q$ and $q+1$ with a travel time of $t_{\text {trav }, q}=\frac{1.3 \cdot d_{\text {trav }, q}}{4.0 \mathrm{~km} / \mathrm{h}}$, where $d_{\text {trav }, q}$ is the beeline distance between the two activities.

Travel times for all other transport modes are approximated by congested car travel times (for colectivo, other, ride, taxi) or by teleportation similar to the walk mode (bike, train) with different

\footnotetext{
3 See http://datos.gob.cl/dataset/1587, accessed 13 August 2015.

${ }^{4}$ See https://svn.vsp.tu-berlin.de/repos/public-svn/matsim/scenarios/countries/cl/santiago/ or search from http://matsim.org/datasets.

${ }^{5}$ Currently, see https://github.com/matsim-org/matsim/tree/master/playgrounds/santiago/src/main/java/ playground/santiago.
} 
teleportation speeds (10.0 and $50.0 \mathrm{~km} / \mathrm{h}$, respectively). Monetary costs are also approximated. However, as long as switching from/to these modes is not allowed (see next paragraph), this essentially has no effect on simulation results.

\subsubsection{Calibration/validation}

The Alternative Specific Constants of the different modes are determined in the calibration process. The procedure to run the first simulation with 200 iterations, together with the calibration of the constants is explained in Kickhöfer et al. (2016).

Another standard verification of MATSim simulation output is the comparison of traffic flows to data from real-world counting stations. 49 counting stations are available within the Santiago greater area, 40 on major roads, 9 on (parallel) local roads. The counts data is recorded in July 2011. After cleaning the data, 36 counting stations remain with data from 6:00 am to $11: 30 \mathrm{pm}$ in 15 minutes time bins. MATSim traffic output versus observed traffic is analyzed in Kickhöfer et al. (2016), which indicates the need for further calibration efforts once the population is expanded to a $10 \%$ or $100 \%$ sample.

\subsection{Conclusion and Outlook}

This section summarized a MATSim scenario set up from input data that is open and publicly available. This makes the scenario an interesting tool for transparent decision making of public administrations, for advancing transport modeling and policy research as well as for stimulating innovation activity of the private sector. Possible applications include the (economic) evaluation of planned transport policies and projects and the development of business ideas based on the simulated mobility of individuals in Santiago. A number of future model improvements to be implemented in the scenario are provided in Kickhöfer et al. (2016). A non-exhaustive list of potential research problems to be analyzed with the MATSim Santiago scenario is the following:

- the effects of road pricing strategies on travel times, traffic volumes, public transport and demand for non-motorized mobility, air pollution, noise levels, etc.,

- the introduction of alternative interventions such as (full or partial) pedestrianization of the city center, speed limitations, roads with exclusive right-of-way for public transport, platenumber based car driving restrictions, parking restrictions, road closures and road openings, restrictions on truck traffic, new cycleways and new Metro lines, and

- the extraction of accessibility measures to study the land use impacts of transport interventions. 